\title{
INTERNATIONAL JOURNAL OF MATHEMATICS
}

\section{Author Index Volume 12 (2001)}

Adachi, J.,

Generic knots in contact 3manifolds

Agler, J. \& McCarthy, J.E., Interpolating sequences on the bidisk

Álvarez-Cónsul, L. \& GarcíaPrada, O.,

Dimensional reduction, SL(2,C )-equivariant bundles and stable holomorphic chains

Apostolov, V., Drăghici, T. \& Moroianu, A.,

A splitting theorem for Kähler manifolds whose Ricci tensors have constant eigenvalues

Ara, P., Pedersen, G.K. \& Perera, F.,

A closure operation in rings

Bates, T. \& Giordano, T., Bounded cocycles on finite von Neumann algebras

Bolton, J. \& Woodward, L.M., Linearly full harmonic 2-spheres in $S^{4}$ of area $20 \pi$

Boyer, C.P., Hurtubise, J.C. \& Milgram, R.J.,

Stability theorems for spaces of rational curves

Bruasse, L.,

Harder-Narasimhan filtration on non Kähler manifolds

Bursztyn, H. \& Waldmann, S., *-Ideals and formal Morita equivalence of $*$-algebras

Caddeo, R., Montaldo, S. \& Oniciuc, C.,

Biharmonic submanifolds of $\mathrm{S}^{3}$

Chen, B.-Y.,

A note on Bergman completeness

Consani, C. \& Scholten, J., Arithmetic on a quintic threefold

Danilenko, A.I.,

Strong orbit equivalence of locally compact cantor minimal systems

Debalme, R. \& Ivashkovich, S., Complete hyperbolic neighborhoods in almost-complex surfaces
6(2001) 625

9(2001) 1103

2(2001) 159

7(2001) 769

$7(2001) 791$

6(2001) 743

5(2001) 535

2(2001) 223

5(2001) 579

5(2001) 555

8(2001) 867

4(2001) 383

8(2001) 943

1(2001) 113

2(2001) 211
Deicke, K.,

Exterior equivalence for pointwise unitary coactions

Dekimpe, K.,

Cohomology vanishing results and polynomial structures

Demailly, J.-P., Peternell, T. \& Schneider, M.,

Pseudo-effective line bundles on compact Kähler manifolds

Denef, J. \& Loeser, F., Macdonald integrals and monodromy

Doplicher, S., Pinzari, C. \& 4(2001) 415 Roberts, J.E.,

An algebraic duality theory for multiplicative unitaries

Dowker, J.S., Kirsten, K. \& Gilkey, P.B.,

On properties of the asymptotic expansion of the heat trace for the $N / D$ problem

Drăghici, T., see Apostolov

Esnault, H. \& Hertling, C., Semistable bundles on curves and reducible representations of the fundamental group

Fukuhara, S., Matsumoto, Y. \& Yui, N.,

Non-commutative polynomial reciprocity formulae

Futamura, H., Kataoka, N. \& Kishimoto, A.,

Homogeneity of the pure state space for separable $C^{*}$-algebras

García-Prada, O., see Álvarez-Cónsul

Gilkey, P.B., see Dowker

Giordano, T. see Bates

Guido, D. \& Isola, T., A semicontinuous trace for almost local operators on an open manifold

Hamana, M., Infinite, $\sigma$-finite, non- $W^{*}, A W^{*}$ factors
1(2001) 63

2(2001) 143

6(2001) 689

9(2001) 987

5(2001) 505

7(2001) 769

7(2001) 847

8(2001) 973

7(2001) 813

2(2001) 159

5(2001) 505

6(2001) 743

9(2001) 1087

1(2001) 81 
Haworth, P.A., Local characterization of approximately finite operator algebras

Hertling, C., see Esnault

Holla, Y.I. \& Nitsure, N., Topology of quadric bundles

Hurtubise, J.C., see Boyer

Hyeon, D., Higgs bundles, spectral curves and étale covering

Ishii, S. \& Prokhorov, Y., Hypersurface exceptional singularities

Isola, $\mathrm{T}$., see Guido

Ivashkovich, S., see Debalme

Iyer, J.N.,

Line bundles of type $(1, \ldots, 1,2$, $\ldots, 2,4, \ldots 4)$ on Abelian varieties

Kataoka, N., see Futamura

Kirsten, K. see Dowker

Kishimoto, A., see Futamura

Kodiyalam, V. \& Sunder, V.S., Spectra of principal graphs

Koike, K., Shiga, H., Takayama, N. \& Tsutsui, T.,

Study on the family of $K 3$ surfaces induced from the lattice $\left(D_{4}\right)^{3} \oplus\langle-2\rangle \oplus\langle 2\rangle$

Kustermans, J., Locally compact quantum groups in the universal setting

Loeser, F. see Denef

Lu, Z.Q.,

On the lower bound estimates of sections of the canonical bundles over a Riemann surface

Mai, N.T.T. see Thai

Masuda, T.

Extension of automorphisms of a subfactor to the symmetric enveloping algebra

Matsumoto, Y., see Fukuhara

McCarthy, J.E., see Agler
$7(2001) 751$

7(2001) 847

9(2001) 1005

2(2001) 223

4(2001) 393

6(2001) 661

9(2001) 1087

2(2001) 211

1(2001) 125

7(2001) 813

5(2001) 505

7(2001) 813

2(2001) 203

9(2001) 1049

3(2001) 289

9(2001) 987

8(2001) 891

7(2001) 857

6(2001) 637

8(2001) 973

9(2001) 1103
Milgram, R.J., see Boyer

Montaldo, S., see Caddeo

Moriwaki, A., The Q -picard group of the moduli space of curves in positive characteristic

Moroianu, A., see Apostolov

Mukai-Hidano, M. \& Ohnita, Y., Geometry of the moduli spaces of harmonic maps into Lie groups via gauge theory over Riemann surfaces

Nilsen, M.M. \& Smith, R.R., Approximation properties for crossed products by actions and coactions

Nitsure, N. see Holla

Ohnita, Y., see Mukai-Hidano

Okuma, T., Simultaneous good resolutions of deformations of Gorenstein surface singularities

Oniciuc, C., see Caddeo

Pask, D. \& Raeburn, I., Symmetric imprimitivity theorems for graph $C^{*}$-algebras

Pedersen, G.K., see Ara

Perera, F., see Ara

Peternell, T., see Demailly

Phillips, N.C., Continuous-trace $C^{*}$-algebras not isomorphic to their opposite algebras

Pinzari, C. see Doplicher

Pisier, G. Remarks on the similarity degree of an operator algebra

Prokhorov, Y., see Ishii

Raeburn, I., see Pask

Roberts, J.E., see Doplicher

Schneider, M. see Demailly
2(2001) 223

8(2001) 867

5(2001) 519

$7(2001) 769$

3(2001) 339

5(2001) 595

9(2001) 1005

3(2001) 339

1(2001) 49

8(2001) 867

5(2001) 609

7(2001) 791

7(2001) 791

6(2001) 689

3(2001) 263

4(2001) 415

4(2001) 403

6(2001) 661

5(2001) 609

4(2001) 415

6(2001) 689 
Schochet, C.L.,

3(2001) 373

Geometric realization and $K$ theoretic decomposition of $C^{*}$ algebras

Scholten, J., see Consani

Shiga, H., see Koike

Smith, R.R., see Nilsen

Sukhov, A. \& Tumanov, A., Stationary discs and geometry of $\mathrm{CR}$ manifolds of codimension two

Sunder, V.S., see Kodiyalam

Takakura, T., Intersection theory on symplectic quotients of products of spheres

Takayama, N., see Koike

Tang, Z.Z., New constructions of eigenmaps between spheres

Tanner, E., see Wilson

Tanner, E., see Wilson

Thai, D.D. \& Mai, N.T.T., Hartogs-type extension theorem and singular sets of separately holomorphic mappings on compact sets with values in a weakly brody hyperbolic complex space
8(2001) 943

9(2001) 1049

5(2001) 595

8(2001) 877

2(2001) 203

1(2001) 97

9(2001) 1049

3(2001) 277

1(2001)1

1(2001) 37

7(2001) 857
Tsutsui, T., see Koike

Tumanov, A., see Sukhov

Waldmann, S.,

9(2001) 1049

8(2001) 877

5(2001) 555

see Bursztyn

Wilson, R. \& Tanner, E.,

1(2001)1

Irreducible unitary representations of $\mathrm{SU}_{p, q} \mathrm{I}$ : The discrete series

Wilson, R. \& Tanner, E.,

Woodward, L.M.,

Woronowicz, S.L.,

Yamada, K.,

Yui, N., Irreducible unitary representations of $\mathrm{SU}_{p, q}$ II: The continuous series see Bolton Quantum ' $a z+b$ ' group on complex plane

A criterion for getting a big component of the moduli of vector bundles by changing a polarization

8(2001) 973

1(2001) 37

5(2001) 535

4(2001) 461

8(2001) 927

see Fukuhara 